\title{
Neutron capture cross section of ${ }^{90} \mathrm{Zr}$ : Bottleneck in the $s$-process reaction flow
}

G. Tagliente,,${ }^{1, *}$ K. Fujii, ${ }^{2}$ P. M. Milazzo,${ }^{2}$ C. Moreau, ${ }^{2}$ G. Aerts, ${ }^{3}$ U. Abbondanno, ${ }^{2}$ H. Álvarez,${ }^{4}$ F. Alvarez-Velarde, ${ }^{5}$ S. Andriamonje, ${ }^{3}$ J. Andrzejewski, ${ }^{6}$ P. Assimakopoulos, ${ }^{7}$ L. Audouin,,${ }^{8}$ G. Badurek,${ }^{9}$ P. Baumann, ${ }^{10}$ F. Bečváŕ, ${ }^{11}$ E. Berthoumieux ${ }^{3}$ S. Bisterzo, ${ }^{12}$ F. Calviño, ${ }^{13}$ M. Calviani, ${ }^{14}$ D. Cano-Ott,${ }^{5}$ R. Capote $,{ }^{15},{ }^{16}$ C. Carrapiço, ${ }^{17}$ P. Cennini, ${ }^{18}$ V. Chepel, ${ }^{19}$ E. Chiaveri, ${ }^{18}$ N. Colonna,,${ }^{1}$ G. Cortes, ${ }^{13}$ A. Couture, ${ }^{20}$ J. Cox,${ }^{20}$ M. Dahlfors, ${ }^{18}$ S. David,${ }^{10}$ I. Dillman, ${ }^{8}$

C. Domingo-Pardo, ${ }^{21}$ W. Dridi, ${ }^{3}$ I. Duran, ${ }^{4}$ C. Eleftheriadis,${ }^{22}$ M. Embid-Segura, ${ }^{5}$ L. Ferrant, ${ }^{23}$ A. Ferrari, ${ }^{18}$

R. Ferreira-Marques, ${ }^{19}$ W. Furman ${ }^{24}$ R. Gallino, ${ }^{12}$ I. Goncalves ${ }^{19}$ E. Gonzalez-Romero, ${ }^{5}$ F. Gramegna, ${ }^{14}$ C. Guerrero,${ }^{5}$ F. Gunsing, ${ }^{3}$ B. Haas, ${ }^{25}$ R. Haight, ${ }^{26}$ M. Heil,${ }^{8}$ A. Herrera-Martinez, ${ }^{18}$ M. Igashira, ${ }^{27}$ E. Jericha, ${ }^{9}$ F. Käppeler,${ }^{8}$ Y. Kadi, ${ }^{18}$ D. Karadimos, ${ }^{7}$ D. Karamanis, ${ }^{7}$ M. Kerveno,${ }^{10}$ P. Koehler ${ }^{28}$ E. Kossionides, ${ }^{29}$ M. Krtička, ${ }^{11}$ C. Lamboudis,${ }^{22}$ H. Leeb,${ }^{9}$ A. Lindote,${ }^{19}$ I. Lopes,${ }^{19}$ M. Lozano, ${ }^{16}$ S. Lukic,${ }^{10}$ J. Marganiec, ${ }^{6}$ S. Marrone, ${ }^{1}$ T. Martínez,${ }^{5}$ C. Massimi, ${ }^{30}$ P. Mastinu, ${ }^{14}$ A. Mengoni, ${ }^{18,31}$ M. Mosconi,${ }^{8}$ F. Neves,${ }^{19}$ H. Oberhummer, ${ }^{9}$ S. O'Brien, ${ }^{20}$ J. Pancin, ${ }^{3}$ C. Papachristodoulou, ${ }^{7}$

C. Papadopoulos, ${ }^{32}$ C. Paradela, ${ }^{4}$ N. Patronis, ${ }^{7}$ A. Pavlik, ${ }^{33}$ P. Pavlopoulos,${ }^{34}$ L. Perrot,${ }^{3}$ M. T. Pigni, ${ }^{9}$ R. Plag, ${ }^{8}$ A. Plompen, ${ }^{35}$ A. Plukis, ${ }^{3}$ A. Poch ${ }^{13}$ J. Praena, ${ }^{14}$ C. Pretel, ${ }^{13}$ J. Quesada, ${ }^{16}$ T. Rauscher,${ }^{36}$ R. Reifarth, ${ }^{26}$ C. Rubbia, ${ }^{37}$ G. Rudolf, ${ }^{10}$ P. Rullhusen, ${ }^{35}$ J. Salgado, ${ }^{17}$ C. Santos,${ }^{17}$ L. Sarchiapone, ${ }^{18}$ I. Savvidis, ${ }^{22}$ C. Stephan, ${ }^{23}$ J. L. Tain, ${ }^{21}$ L. Tassan-Got, ${ }^{23}$ L. Tavora, ${ }^{17}$ R. Terlizzi, ${ }^{1}$ G. Vannini, ${ }^{30}$ P. Vaz, ${ }^{17}$ A. Ventura, ${ }^{38}$ D. Villamarin, ${ }^{5}$ M. C. Vincente, ${ }^{5}$ V. Vlachoudis ${ }^{18}$ R. Vlastou, ${ }^{32}$ F. Voss, ${ }^{8}$ S. Walter, ${ }^{8} \mathrm{H}$. Wendler, ${ }^{18} \mathrm{M}$. Wiescher ${ }^{20}$ and K. Wisshak ${ }^{8}$ (n_TOF Collaboration ${ }^{\dagger}$ )

${ }^{1}$ Istituto Nazionale di Fisica Nucleare (INFN), Bari, Italy

${ }^{2}$ Istituto Nazionale di Fisica Nucleare (INFN), Trieste, Italy

${ }^{3}$ CEA/Saclay-DSM/DAPNIA, Gif-sur-Yvette, France

${ }^{4}$ Universidade de Santiago de Compostela, Spain

${ }^{5}$ Centro de Investigaciones Energeticas Medioambientales y Technologicas, Madrid, Spain

${ }^{6}$ University of Lodz, Lodz, Poland

${ }^{7}$ University of Ioannina, Greece

${ }^{8}$ Forschungszentrum Karlsruhe GmbH (FZK), Institut für Kernphysik, Germany

${ }^{9}$ Atominstitut der Österreichischen Universitäten, Technische Universität Wien, Austria

${ }^{10}$ Centre National de la Recherche Scientifique/IN2P3-IReS, Strasbourg, France

${ }^{11}$ Univerzita Karlova v Praze, Czech Republic

${ }^{12}$ Dipartimento di Fisica Generale, Università di Torino, Italy

${ }^{13}$ Universitat Politecnica de Catalunya, Barcelona, Spain

${ }^{14}$ Istituto Nazionale di Fisica Nucleare (INFN), Laboratori Nazionali di Legnaro, Italy

${ }^{15}$ International Atomic Energy Agency, NAPC/Nuclear Data Section, Vienna, Austria ${ }^{16}$ Universidad de Sevilla, Spain

${ }^{17}$ Instituto Tecnológico e Nuclear (ITN), Lisbon, Portugal ${ }^{18}$ CERN, Geneva, Switzerland

${ }^{19}$ LIP-Coimbra \& Departamento de Fisica da Universidade de Coimbra, Portugal

${ }^{20}$ University of Notre Dame, Notre Dame, Indiana 46556, USA

${ }^{21}$ Instituto de Física Corpuscular, CSIC-Universidad de Valencia, Spain

${ }^{22}$ Aristotle University of Thessaloniki, Greece

${ }^{23}$ Centre National de la Recherche Scientifique/IN2P3-IPN, Orsay, France

${ }^{24}$ Joint Institute for Nuclear Research, Frank Laboratory of Neutron Physics, Dubna, Russia

${ }^{25}$ Centre National de la Recherche Scientifique/IN2P3-CENBG, Bordeaux, France

${ }^{26}$ Los Alamos National Laboratory, Los Alamos, New Mexico 87545, USA

${ }^{27}$ Tokyo Institute of Technology, Tokyo, Japan

${ }^{28}$ Oak Ridge National Laboratory, Physics Division, Oak Ridge, Tennessee 37831, USA

${ }^{29}$ NCSR, Athens, Greece

${ }^{30}$ Dipartimento di Fisica, Università di Bologna, and Sezione INFN di Bologna, Italy

${ }^{31}$ International Atomic Energy Agency (IAEA), NAPC/Nuclear Data Section, Vienna, Austria

${ }^{32}$ National Technical University of Athens, Greece

${ }^{33}$ Institut für Fakultät für Physik, Universität Wien, Austria

${ }^{34}$ Pôle Universitaire Léonard de Vinci, Paris La Défense, France ${ }^{35}$ CEC-JRC-IRMM, Geel, Belgium

${ }^{36}$ Department of Physics and Astronomy, University of Basel, Basel, Switzerland

${ }^{37}$ Università degli Studi di Pavia, Pavia, Italy

${ }^{38}$ ENEA, Bologna, Italy

(Received 21 December 2007; published 11 March 2008) 
The neutron capture cross sections of the $\mathrm{Zr}$ isotopes have important implications in nuclear astrophysics and for reactor design. The small cross section of the neutron magic nucleus ${ }^{90} \mathrm{Zr}$, which accounts for more than $50 \%$ of natural zirconium represents one of the key isotopes for the stellar $s$-process, because it acts as a bottleneck in the neutron capture chain between the Fe seed and the heavier isotopes. The same element, $\mathrm{Zr}$, also is an important component of the structural materials used in traditional and advanced nuclear reactors. The $(n, \gamma)$ cross section has been measured at CERN, using the $n_{-}$TOF spallation neutron source. In total, 45 resonances could be resolved in the neutron energy range below $70 \mathrm{keV}, 10$ being observed for the first time thanks to the high resolution and low backgrounds at $\mathrm{n}_{-}$TOF. On average, the $\Gamma_{\gamma}$ widths obtained in resonance analyses with the $R$-matrix code SAMMY were $15 \%$ smaller than reported previously. By these results, the accuracy of the Maxwellian averaged cross section for $s$-process calculations has been improved by more than a factor of 2 .

DOI: 10.1103/PhysRevC.77.035802

PACS number(s): 25.40.Lw, 25.70.Ef, 27.60.+j, 97.10.Cv

\section{INTRODUCTION}

Elements heavier than iron are produced mainly via neutron capture reactions by two dominant processes, which are named for their very different time scales: the rapid $(r)$ process related to extremely hot $\left(T>10^{9} \mathrm{~K}\right)$ and neutronrich $\left(n_{n} \gg 10^{20} \mathrm{~cm}^{-3}\right)$ environments with neutron capture times of milliseconds and the slow $s$ process that occurs at lower temperatures $\left[T \approx(1-3) \times 10^{8} \mathrm{~K}\right]$ and neutron densities $\left(n_{n} \approx 10^{8}-10^{10} \mathrm{~cm}^{-3}\right)$ with typical capture times of about a year. The terms slow and rapid refer to the average $\beta$-decay half-lives close to the valley of stability. Approximately one half of the abundances in the mass region $A \geqslant 56$ can be assigned to each of the two processes.

The focus of this article is on the $s$ process. Because of the slow time scale for neutron capture, the reaction chain of subsequent neutron captures and $\beta$ decays follows the valley of $\beta$ stability. The abundances produced in the $s$-process are essentially determined by the neutron capture cross sections of the involved isotopes. The stellar neutron capture rate is given by the neutron capture cross section $\sigma\left(E_{n}\right)$ integrated over the neutron velocity distribution, $\langle\sigma v\rangle=\int_{0}^{\infty} \sigma v \Phi(v) d v$. The velocity distribution $\Phi(v)$ of the neutron flux is described by a Maxwell-Boltzmann form [1], because neutrons are quickly thermalized in the He burning stellar plasma, where the $s$ process takes place.

The $s$ abundance of a stable isotope of mass $A$ is determined by the respective rates for production and destruction:

$$
\frac{d N_{s}(A)}{d t}=n_{n}\langle\sigma v\rangle_{A-1} N_{A-1}-n_{n}\langle\sigma v\rangle_{A} N_{A},
$$

where $n_{n}$ is the neutron density. The term $\langle\sigma v\rangle_{A}$ can be expressed as $\sigma_{A} v_{T}$ by introducing the Maxwellian-averaged capture cross section (MACS) integrated over the stellar spectrum and using the mean thermal neutron velocity $v_{T}$. If one defines the neutron exposure by $\tau=\int n_{n} v_{T} d t$, Eq. (1) can be rewritten as

$$
\frac{d N_{A}}{d \tau}=-\sigma_{A} N_{A}+\sigma_{A-1} N_{A-1} .
$$

In a steady-state situation with $d N_{A} / d \tau=0$, the product $\sigma_{A} N_{A}$ is constant.

\footnotetext{
*giuseppe.tagliente@ba.infn.it

†URL:www.cern.ch/ntof
}

The abundance distribution shows, indeed, that $\sigma_{A} N_{A}$ is roughly constant in mass regions between magic neutron numbers $(50,82,126)$, thus indicating that flow equilibrium was partially reached. The very small neutron capture cross sections of neutron magic isotopes, however, act as bottlenecks for the reaction flow and give rise to steplike discontinuities in the $\sigma_{A} N_{A}$ distribution. This is illustrated qualitatively in Fig. 1, which shows the distribution (normalized with respect to the silicon abundance) as a function of neutron number for the prominent $s$-only isotopes in the reaction path. Solar system abundances and MACS are from Refs. [2] and [1], respectively. The effect of magic neutron numbers is emphasized by vertical lines to indicate the corresponding bottleneck effect. The size of this effect is determined by the time-integrated neutron flux but depends also critically on the smallness of the $(n, \gamma)$ cross sections of the neutron magic isotopes. Because ${ }^{90} \mathrm{Zr}$ is situated at magic number $N=50$, its cross section contributes to the first bottleneck in the reaction flow from the Fe seed toward heavier isotopes (Fig. 1).

In an astrophysical context, the mass region around $N=$ 50 is particularly interesting. Massive stars, where the $s$ process takes place during the presupernova evolution, i.e., during convective core $\mathrm{He}$ burning and convective carbon shell burning [3], contribute only to the abundances below $A=90$. This so-called weak $s$ process is complemented by the main $s$ component in asymptotic giant branch (AGB) stars of $1 \leqslant M / M_{\odot} \leqslant 3$ (where $M_{\odot}$ denotes the mass of the Sun), which is producing the $s$-abundances above $A=90$ [4-6]. Accordingly, ${ }^{90} \mathrm{Zr}$ plays a key role in understanding the situation at the matching point of both components.

Another important aspect is related to the interpretation of the isotopic pattern of $\mathrm{Zr}$ observed in presolar grains [7], which provides a test for the $s$-process efficiency due to the ${ }^{13} \mathrm{C}$ neutron source reaction.

Experimental cross sections with uncertainties $\leqslant 3 \%$ are necessary for such investigations as well as for comparisons between different AGB models [8]; data of this quality are not available today [1]. Especially the status of the ${ }^{90} \mathrm{Zr}$ cross section is not satisfactory, because different measurements have reported uncertainties between 6 and $25 \%$ but exhibit discrepancies up to a factor of 2 [9-15].

From the point of view of nuclear technology, zirconium is widely used as constituent of cladding materials for fuel elements. The low neutron capture cross sections of the $\mathrm{Zr}$ isotopes lead to small activation and in combination with 


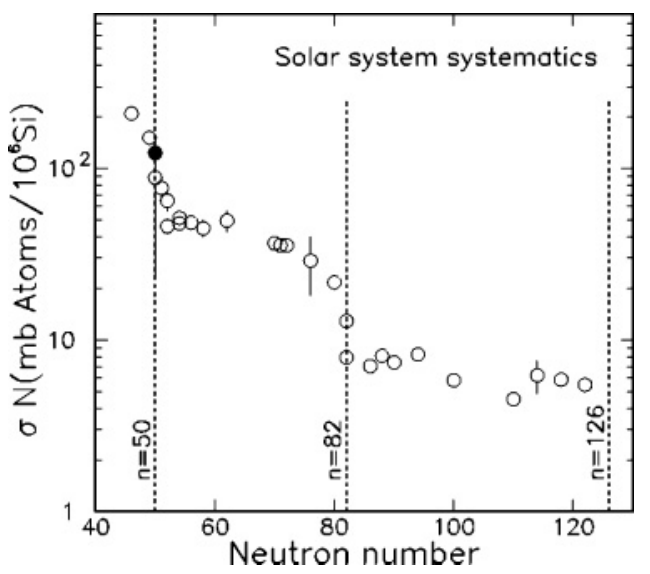

FIG. 1. The product of Maxwellian averaged cross sections and solar abundances (on the silicon $=10^{6} \mathrm{scale}$ ) of prominent $s$-only isotopes as a function of the neutron number. The bottleneck effect at magic neutron numbers is clearly seen even in this rather schematic plot. The full dot refers to ${ }^{90} \mathrm{Zr}$.

favorable chemical and mechanical properties it is best suited for claddings. Accordingly, improved measurements of the $\mathrm{Zr}$ cross section are part of the High Priority Nuclear Data Request List compiled by the Nuclear Energy Agency (NEA/OECD) [16].

Motivated by these requirements, the $\mathrm{Zr}$ cross sections were remeasured at the $\mathrm{n}_{-} \mathrm{TOF}$ facility at CERN using carefully designed experimental techniques and analysis tools as described. The resulting MACS for ${ }^{90} \mathrm{Zr}$ and a brief account of the astrophysical implications are presented.

\section{EXPERIMENTAL SETUP}

\section{A. The $\mathbf{n}_{-}$TOF facility}

The measurements were performed in the energy range between $1 \mathrm{eV}$ and $1 \mathrm{MeV}$ using the pulsed neutron beam of the n_TOF facility at CERN, which is generated by proton-induced spallation reactions on a massive lead target [17]. The main features of the proton beam are its high energy $\left(E_{p}=20 \mathrm{GeV}\right)$, high intensity $\left(I_{p}=7 \times 10^{12}\right.$ protons per bunch), short pulse width ( $\Delta t=6 \mathrm{~ns}$ ), and extremely low duty factor (repetition rate $0.8 \mathrm{~Hz}$ ). The low repetition rate of the proton beam, the high instantaneous neutron flux, and the favorable background conditions in the experimental area make this facility unique for high-resolution time-of-flight (TOF) measurements of neutron-induced reaction cross sections [18].

The spallation neutrons are slowed down in the lead target and moderated in a $5.8 \mathrm{~cm}$-thick layer of cooling water surrounding the target. The resulting neutron spectrum runs from $1 \mathrm{eV}$ up to $250 \mathrm{MeV}$ with a nearly $1 / E$ isolethargic flux up to $1 \mathrm{MeV}$.

The neutron beam is transported through an evacuated flight path with collimators at 135 and $175 \mathrm{~m}$ to the measuring station at a distance of $185.2 \mathrm{~m}$ from the spallation target. The beam line extends $12 \mathrm{~m}$ beyond the experimental area to minimize the effect of back-scattered neutrons. Background due to fast charged particles is suppressed by a 1.5 -T sweeping magnet, heavy concrete walls, and a $3.5 \mathrm{~m}$-thick iron shielding [18].

\section{B. Capture detectors and flux measurement}

The measurement was carried out by detection of the prompt $\gamma$-ray cascade following neutron capture. Two $\gamma$ detectors, consisting of $\mathrm{C}_{6} \mathrm{D}_{6}$ liquid scintillator cells, were placed perpendicular to the neutron beam at a distance of about $3 \mathrm{~cm}$ from the beam axis. In this position the neutron beam was $1.8 \mathrm{~cm}$ in diameter (FWHM). Background due to in-beam $\gamma$-rays from $(n, \gamma)$ reactions in the water moderator [18] was reduced by placing the detectors $9.2 \mathrm{~cm}$ upstream of the sample position. A schematic sketch of the experimental setup is shown in Fig. 2 of Ref. [19].

Special care was devoted to reduce the neutron sensitivity of the experimental setup to the smallest possible level. This was achieved by detailed Monte Carlo simulations of all detector components and with a reduction of materials around the scintillator cells. In this way, an optimized detector was developed for accurate measurements of the resonancedominated $(n, \gamma)$ cross sections of light and neutron magic isotopes [20].

The scintillator cells were directly coupled to EMI9823QKB photomultiplier tubes. The light output of the detectors was calibrated at 662,1173 , and $1332 \mathrm{keV}$ by means of ${ }^{137} \mathrm{Cs}$ and ${ }^{60} \mathrm{Co}$ reference sources. An additional calibration point at $6.13 \mathrm{MeV}$ was obtained by means of a composite ${ }^{238} \mathrm{Pu} /{ }^{13} \mathrm{C}$ source. The calibration was repeated in regular intervals to verify the stability of the detectors and of the data acquisition system.

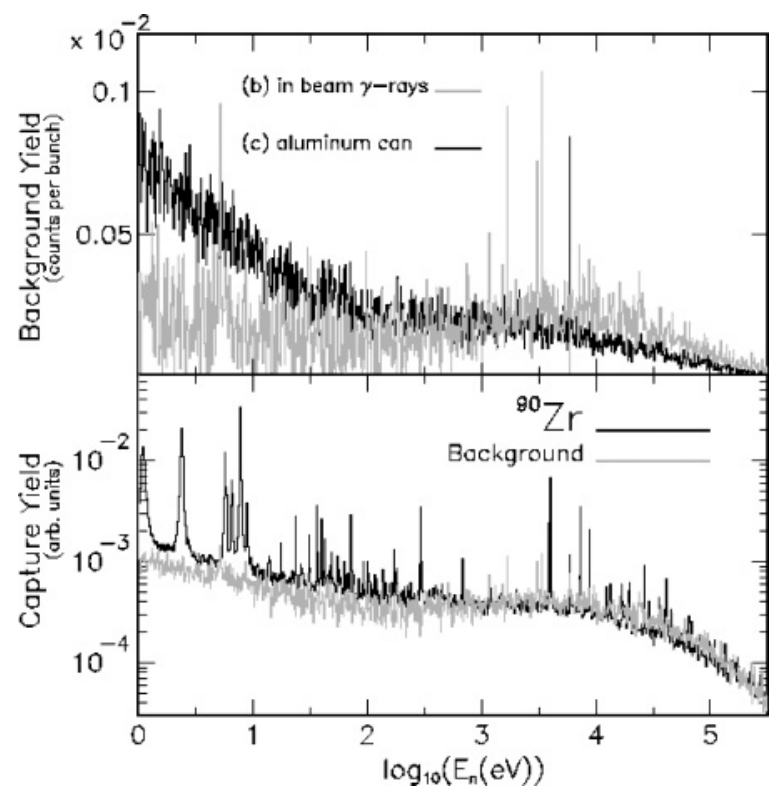

FIG. 2. (Upper panel) Main background components due to inbeam $\gamma$ rays (gray) and to capture events in the aluminum can of the $\mathrm{Zr}$ sample (black), as a function of the energy of incident neutrons (En). (Lower panel) Capture yield of ${ }^{90} \mathrm{Zr}$ (black) and overall background (gray), as a function of the energy of incident neutrons (En). The resonances below neutron energy of $3.8 \mathrm{keV}$ are due to $\mathrm{Hf}$ and $\mathrm{Sn}$ contaminations in the sample. 
The detector signals were recorded using the standard $\mathrm{n}_{-} \mathrm{TOF}$ data acquisition system based on fast digitizers with a sampling rate of $500 \mathrm{MSamples} / \mathrm{s}$ [21]. The neutron energy related to each event was inferred from the TOF that was measured relative to the time stamp defined by the impact of the proton pulse.

The relative neutron flux was measured with a low mass flux monitor consisting of a Mylar foil, $1.5 \mu \mathrm{m}$ in thickness, with a layer of $200 \mu \mathrm{g} / \mathrm{cm}^{2}$ of ${ }^{6} \mathrm{Li}$. Charged particles emitted in ${ }^{6} \mathrm{Li}(n, \alpha)^{3} \mathrm{H}$ reactions were detected by a set of four $\mathrm{Si}$ detectors surrounding the sample outside the neutron beam [22]. The monitor sample was located $1.5 \mathrm{~m}$ upstream of the capture samples. The neutron beam was also periodically checked by calibration runs with gold reference samples.

\section{Samples}

The ${ }^{90} \mathrm{Zr}$ sample was prepared from $\mathrm{ZrO}_{2}$ powder with an enrichment of $97.7 \%$. The oxide powder was pressed to a pellet $22 \mathrm{~mm}$ in diameter, $1.3 \mathrm{~mm}$ in thickness, and $2.717 \mathrm{~g}$ in mass. This pellet was then encapsulated in a very thin aluminum can with a total weight of about $300 \mathrm{mg}$. Traces of Hf, Sn, $\mathrm{Na}, \mathrm{Mg}$, and $\mathrm{Al}$ were also present in the sample. Although these impurities were below $0.01 \%$, their contribution to the measured capture yield was not negligible and had to be considered in the data analysis. For example, the significant resonances below the first ${ }^{90} \mathrm{Zr}$ resonance at $3.86 \mathrm{keV}$ (Fig. 2) must be attributed to contaminating $\mathrm{Hf}$ and $\mathrm{Sn}$ isotopes, whose resonance distributions are well known.

Additional $\mathrm{Au}$ and $\mathrm{Pb}$ samples of the same diameter were used for repeated neutron flux measurements and background runs throughout the experiment. The relevant sample characteristics are summarized in Table I.

\section{DETERMINATION OF CAPTURE YIELDS}

The quantity to be determined in a neutron capture experiment is the yield, which is defined as the fraction of incident neutrons undergoing $(n, \gamma)$ reactions in the sample. The capture yield is directly linked to the capture and the total cross sections. Because the capture detectors have low efficiency and cover a restricted solid angle far below $4 \pi$, the overall efficiency for the detection of capture events was $\approx 20 \%$. For the determination of the absolute yield, the measured yield has to be normalized to the standard cross section of a reference isotope.

This normalization was performed by means of the saturated resonance technique [23] using the $4.9 \mathrm{eV}$ resonance in

TABLE I. Sample characteristics.

\begin{tabular}{lccccccc}
\hline \hline Sample & $\begin{array}{c}\text { Chemical } \\
\text { form }\end{array}$ & \multicolumn{4}{c}{ Isotopic composition (\%) } & \multirow{2}{*}{$\begin{array}{c}\text { Thickness } \\
\text { (atoms } / \mathrm{b})\end{array}$} \\
\cline { 3 - 6 } & & ${ }^{90} \mathrm{Zr}$ & ${ }^{91} \mathrm{Zr}$ & ${ }^{92} \mathrm{Zr}$ & ${ }^{94} \mathrm{Zr}$ & ${ }^{96} \mathrm{Zr}$ & \\
\hline${ }^{90} \mathrm{Zr}$ & $\mathrm{ZrO}_{2}$ & 97.7 & 0.87 & 0.60 & 0.67 & 0.16 & 0.003531 \\
${ }^{n a t} \mathrm{~Pb}$ & Metal & & & & & & 0.002992 \\
${ }^{197} \mathrm{Au}$ & Metal & & & & & & 0.001498 \\
\hline \hline
\end{tabular}

${ }^{197} \mathrm{Au}$. For this calibration method it is essential to determine the energy dependence of the neutron flux with good accuracy as described below.

\section{A. Pulse height weighting technique}

Neutron capture events are characterized by the prompt $\gamma$-ray cascade emitted from the compound nucleus. Because the corresponding $\gamma$-ray spectrum depends on the isotope under study and may even change from resonance to resonance for a given isotope, the capture detectors have to satisfy certain criteria. In the case of ${ }^{90} \mathrm{Zr}$, another difficulty comes from the large scattering/capture ratio expected for this neutron magic nucleus. Especially with respect to the second point, $\mathrm{C}_{6} \mathrm{D}_{6}$ detectors have been chosen for the present experiment. These detectors have (i) sufficiently low efficiency to detect at most one $\gamma$-ray per cascade, which makes them suitable for the pulse height weighting technique (PHWT), and (ii) are practically insensitive to scattered neutrons [20].

To detect capture events independent of the cascade multiplicity, the intrinsic efficiency of $\mathrm{C}_{6} \mathrm{D}_{6}$ detectors has to be corrected such that it is linearly increasing with $\gamma$-ray energy. In this case, the efficiency for capture cascades is proportional to the total released $\gamma$ energy, independent of multiplicity and of the $\gamma$ spectrum. The required proportionality is achieved via the PHWT, an off-line modification of the response function of the detector, which has been extensively studied for the setup used at n_TOF [24-26].

The determination of the weighting functions was performed by means of detailed Monte Carlo simulations for the entire detector setup, following the prescriptions outlined in Ref. [26].

\section{B. Neutron flux determination}

The neutron flux at $\mathrm{n}_{-}$TOF has been measured with a ${ }^{235} \mathrm{U}$ loaded parallel plate fission chamber from PTB Braunschweig [17] (see also Fig. 2 of Ref. [27]) as well as with the ${ }^{6} \mathrm{Li}$ neutron flux monitors used during the experimental runs [22]. Additional flux measurements were performed using parallel plate avalanche counters [28] and by the analysis of standard resonances [29]. In this way the neutron flux could be determined with an overall uncertainty of $2 \%$.

\section{Background components}

Background contributions in neutron capture measurements at $\mathrm{n}_{-} \mathrm{TOF}$ are mainly due to (i) capture events in the detectors or in surrounding materials due to neutrons scattered from the sample; (ii) in-beam $\gamma$-rays produced in the spallation target, which are partly time-correlated with the neutron beam; (iii) capture events in the aluminum can of the $\mathrm{Zr}$ sample; and (iv) the ambient background in the experimental area.

(i) The part due to sample scattered neutrons was estimated by means of a natural carbon sample, where the total cross section is completely dominated by the elastic-scattering channel. The 
effect of scattered neutrons turned out to be negligible in the $\mathrm{Zr}$ spectra, thanks to the very low neutron sensitivity of the setup and because materials with large capture cross sections were removed from the experimental area.

(ii) Scattering of in-beam $\gamma$-rays in the sample appeared to represent the main source of background. This effect scales with atomic number and was, therefore, studied by means of a lead sample. Lead was particularly suited because the capture cross section for lead is very small. The background from in-beam $\gamma$-rays is strongest in the neutron energy range between 1 and $100 \mathrm{keV}$, because $\gamma$ rays from neutron capture in the water moderator around the spallation target arrive at the experimental area in the corresponding TOF interval.

(iii) and (iv) Measurements with an empty Al can and without any sample showed that the related background was smaller but not negligible compared to that from in-beam $\gamma$ rays. However, the ambient background was found to contribute much less, resulting in a negligible uncertainty of this background correction.

The two strongest background sources are compared in the upper panel of Fig. 2, whereas the overall background is plotted in the lower panel.

\section{Discussion of uncertainties}

The most important uncertainty is due to the limited counting statistics. This problem is a direct consequence of the relatively low cross section of ${ }^{90} \mathrm{Zr}$, but partly it is also due to limitations in beam time and to the restricted sample mass. The intention behind the latter aspect was to avoid extensive corrections due to sample related effects such as multiple scattering and neutron and $\gamma$-ray self-shielding. Because of the decreasing signal/background ratio, the statistical uncertainty grows with neutron energy from $\approx 4 \%$ at $3 \mathrm{keV}$ to $\approx 8 \%$ at $70 \mathrm{keV}$. Beyond $70 \mathrm{keV}$, neutron resonance analyses are challenged by counting statistics.

The application of the PHWT implies a systematic uncertainty associated with the Monte Carlo simulations for determining the weighting functions and with the polynomial fit of the calculated data points. Comparison of weighting functions obtained with different combinations of parameters and fit procedures showed that the related uncertainty is less than $2 \%$ [26].

A further source of uncertainty are the energy dependence of the neutron flux and the fraction of the neutron beam covered by the sample. The total uncertainty associated with these contributions was determined by the comparison of various techniques with the saturated resonance technique using the $4.9 \mathrm{eV}$ resonance of the gold. Thus a value of $2 \%$ was obtained.

\section{RESONANCE ANALYSIS}

The experimental yield

$$
Y^{\exp }=\frac{Y_{w}}{N_{n} E_{c}}+B
$$

is determined by the weighted net count rate, $Y_{w}$, the effective binding energy, $E_{c}$, the integrated neutron flux, $N_{n}$, and the overall background, $B$.

Resonances observed in the neutron energy range from 3 to $70 \mathrm{keV}$ were analyzed in the Reich-Moore approximation with the $R$-matrix code SAMMY [30]. The corrections for Doppler broadening of resonance widths due to the thermal motion, for the energy resolution of the neutron beam, for isotopic and chemical sample impurities, and for self-shielding and neutron multiple scattering, are considered in the fits with this code. The Doppler broadening was treated by a free gas model assuming a temperature of $300 \mathrm{~K}$. The effect of potential scattering was calculated using a radius of $7.1 \mathrm{fm}$ [14]. The resolution function of the $\mathrm{n}_{-}$TOF neutron beam becomes important at energies above $1 \mathrm{keV}$ and leads to low energy tails in the resonance shapes.

Among the 45 analyzed resonances, 10 resonances and one doublet around $13.4 \mathrm{keV}$ were identified for the first time [9-13].

The spin assignments in the fits with the SAMMY code were adopted from evaluated parameter sets [14,15]. Because the resonance shape is affected by the spin, these assignments were tested and in all cases confirmed in the analysis. The spins of the newly observed resonances were selected from the quality of the fits.

The neutron widths $\Gamma_{n}$ values listed in the data libraries $[14,15]$, which were derived from transmission measurements $[10,12]$, were adopted as start values in the fits with the possibility to vary within a narrow range, whereas the resonances energies $E_{R}$ and the radiative widths $\Gamma_{\gamma}$ were considered as free fit parameters. Although the resonance energies could be very well defined, the $\Gamma_{\gamma}$ values exhibit uncertainties of $\pm 10 \%$.

The resonance parameters obtained by SAMMY analyses of the background subtracted capture yield are listed in Table II. For each resonance the extracted parameters, i.e., the resonance energy $E_{R}$, the capture width $\Gamma_{\gamma}$, and the neutron width $\Gamma_{n}$, are given together with the capture kernel

$$
K=g \frac{\Gamma_{n} \cdot \Gamma_{\gamma}}{\left(\Gamma_{n}+\Gamma_{\gamma}\right)},
$$

where

$$
g=\frac{(2 J+1)}{\left(2 I_{n}+1\right)\left(2 I_{Z r}+1\right)}
$$

is the statistical spin factor determined by the resonance spin, $J$, the spin of the incident neutron, $I_{n}=1 / 2$, and the spin of the target nucleus, $I_{\mathrm{Zr}}=0$. Examples illustrating the quality of the SAMMY fits are shown in Fig. 3.

The extracted values for $\Gamma_{\gamma}$ are on average $15 \%$ smaller than those reported in a previous measurement [9] as illustrated in Fig. 4. Compared to the evaluated parameter set of Ref. [14], which is based on all available experimental data, our results are approximately $10 \%$ lower.

These systematic differences with respect to data obtained in the pioneering experiments in the mid-1970s $[9,10]$ could possibly be due to smaller corrections (e.g., for self-shielding and multiple scattering), lower backgrounds, and modern data 
TABLE II. Resonance parameters $E_{R}, \Gamma_{n}$, and $\Gamma_{\gamma}$.

\begin{tabular}{|c|c|c|c|c|c|c|c|c|}
\hline$E_{R}(\mathrm{keV})$ & $J$ & $l$ & $\Gamma_{n}(\mathrm{eV})$ & $\Delta \Gamma_{n}(\%)$ & $\Gamma_{\gamma}(\mathrm{eV})$ & $\Delta \Gamma_{\gamma}(\%)$ & $K(\mathrm{eV})$ & $\Delta K(\%)$ \\
\hline $3.8612 \pm 4 \times 10^{-4}$ & $1 / 2$ & 0 & 10.8 & 4.6 & 0.078 & 3.0 & 0.077 & 3.0 \\
\hline $4.00832 \pm 6 \times 10^{-5}$ & $3 / 2$ & 1 & 0.089 & 4.4 & 0.25 & 8.7 & 0.13 & 4.0 \\
\hline $7.2603 \pm 2 \times 10^{-4}$ & $3 / 2$ & 1 & 3.2 & 5.8 & 0.150 & 3.1 & 0.287 & 3.0 \\
\hline $8.8551 \pm 4 \times 10^{-4}$ & $1 / 2$ & 1 & 6.0 & 7.1 & 0.218 & 3.5 & 0.211 & 3.4 \\
\hline $9.60204 \pm 7 \times 10^{-5}$ & $1 / 2$ & 1 & 0.020 & 7.9 & 0.44 & 10.0 & 0.019 & 7.6 \\
\hline $12.20802 \pm 2 \times 10^{-5}$ & $1 / 2$ & 0 & 0.007 & 8.7 & 0.241 & 8.5 & 0.0067 & 9.4 \\
\hline $12.39980 \pm 7 \times 10^{-5 a}$ & $1 / 2$ & 1 & 0.049 & 8.5 & 0.081 & 9.5 & 0.031 & 6.5 \\
\hline $12.44288 \pm 1 \times 10^{-5 a}$ & $1 / 2$ & 1 & 0.046 & 9.1 & 0.057 & 9.3 & 0.026 & 6.6 \\
\hline $13.365 \pm 3 \times 10^{-3 \mathrm{~b}}$ & $1 / 2$ & 0 & 30.0 & 9.2 & 0.068 & 7.2 & 0.068 & 7.9 \\
\hline $13.44390 \pm 1 \times 10^{-5 a}$ & $1 / 2$ & 0 & 53.0 & 9.3 & 0.053 & 6.8 & 0.053 & 8.8 \\
\hline $16.894 \pm 1 \times 10^{-3 \mathrm{a}}$ & $1 / 2$ & 1 & 1.46 & 10.0 & 0.099 & 6.8 & 0.092 & 6.5 \\
\hline $16.92958 \pm 2 \times 10^{-5 a}$ & $1 / 2$ & 1 & 0.026 & 9.6 & 0.279 & 9.4 & 0.024 & 8.4 \\
\hline $16.9792 \pm 1 \times 10^{-4}$ & $1 / 2$ & 0 & 0.020 & 10.0 & 0.246 & 8.7 & 0.018 & 9.3 \\
\hline $17.402 \pm 2 \times 10^{-2}$ & $1 / 2$ & 0 & 241. & 7.3 & 0.162 & 7.5 & 0.162 & 7.5 \\
\hline $19.07930 \pm 1 \times 10^{-5 \mathrm{a}}$ & $1 / 2$ & 1 & 0.105 & 10. & 0.020 & 8.8. & 0.017 & 7.5 \\
\hline $19.098 \pm 2 \times 10^{-3}$ & $1 / 2$ & 1 & 0.70 & 10.0 & 0.11 & 8.0 & 0.097 & 7.1 \\
\hline $19.713 \pm 2 \times 10^{-3}$ & $1 / 2$ & 1 & 13.0 & 9.0 & 0.27 & 5.6 & 0.267 & 5.5 \\
\hline $26.469 \pm 3 \times 10^{-3}$ & $3 / 2$ & 1 & 1.3 & 10. & 0.065 & 8.8 & 0.124 & 8.4 \\
\hline $26.538 \pm 1 \times 10^{-3}$ & $3 / 2$ & 1 & 5.8 & 9.6 & 0.21 & 6.2 & 0.41 & 6.0 \\
\hline $28.827 \pm 3 \times 10^{-3}$ & $3 / 2$ & 1 & 1.1 & 9.8 & 0.15 & 9.8 & 0.27 & 8.8 \\
\hline $35.356 \pm 4 \times 10^{-3}$ & $1 / 2$ & 0 & 38. & 9.4 & 0.59 & 6.3 & 0.579 & 6.2 \\
\hline $39.507 \pm 3 \times 10^{-3}$ & $3 / 2$ & 1 & 1.3 & 10.0 & 0.18 & 8.1 & 0.329 & 7.2 \\
\hline $40.40 \pm 1 \times 10^{-2}$ & $1 / 2$ & 1 & 58. & 10.0 & 0.16 & 9.1 & 0.162 & 9.0 \\
\hline $41.39 \pm 1 \times 10^{-2}$ & $3 / 2$ & 1 & 236. & 7.3 & 0.98 & 5.4 & 1.96 & 5.4 \\
\hline $42.0699 \pm 1 \times 10^{-4 \mathrm{a}}$ & $3 / 2$ & 1 & 0.20 & 10.0 & 0.040 & 10.0 & 0.068 & 8.5 \\
\hline $42.21 \pm 6 \times 10^{-2}$ & $1 / 2$ & 0 & 285. & 9.7 & 0.20 & 9.6 & 0.203 & 9.6 \\
\hline $42.455 \pm 6 \times 10^{-3 \mathrm{a}}$ & $1 / 2$ & 0 & 0.49 & 10.0 & 0.071 & 9.8 & 0.062 & 8.7 \\
\hline $42.64 \pm 2 \times 10^{-2 \mathrm{a}}$ & $1 / 2$ & 0 & 116.0 & 9.6 & 0.20 & 8.7 & 0.20 & 8.7 \\
\hline $44.822 \pm 8 \times 10^{-3}$ & $3 / 2$ & 1 & 83. & 9.6 & 0.31 & 7.2 & 0.627 & 7.2 \\
\hline $53.264 \pm 7 \times 10^{-3 \mathrm{a}}$ & $1 / 2$ & 0 & 0.811 & 10. & 0.025 & 10. & 0.025 & 9.7 \\
\hline $53.371 \pm 8 \times 10^{-3}$ & $1 / 2$ & 0 & 1.5 & 10.0 & 0.094 & 9.8 & 0.088 & 9.2 \\
\hline $53.672 \pm 1 \times 10^{-3}$ & $1 / 2$ & 0 & 0.48 & 10.0 & 0.19 & 10.0 & 0.134 & 7.7 \\
\hline $54.353 \pm 1 \times 10^{-3}$ & $1 / 2$ & 1 & 0.098 & 10.0 & 0.24 & 10.0 & 0.070 & 7.7 \\
\hline $54.546 \pm 1 \times 10^{-3}$ & $1 / 2$ & 1 & 0.22 & 10.0 & 0.25 & 10.0 & 0.117 & 7.1 \\
\hline $55.80 \pm 2 \times 10^{-2}$ & $1 / 2$ & 1 & 64. & 10.0 & 0.19 & 9.3 & 0.192 & 9.3 \\
\hline $56.52 \pm 1 \times 10^{-2}$ & $1 / 2$ & 1 & 41. & 10.0 & 0.25 & 8.8 & 0.244 & 8.7 \\
\hline $57.790 \pm 4 \times 10^{-3}$ & $1 / 2$ & 0 & 0.65 & 9.5 & 0.42 & 9.5 & 0.257 & 6.9 \\
\hline $58.311 \pm 8 \times 10^{-3 \mathrm{a}}$ & $1 / 2$ & 1 & 9.4 & 10.0 & 0.18 & 9.0 & 0.174 & 8.9 \\
\hline $61.9000 \pm 2 \times 10^{-4}$ & $3 / 2$ & 1 & 0.70 & 10.0 & 0.20 & 10.0 & 0.313 & 8.1 \\
\hline $62.410 \pm 2 \times 10^{-3}$ & $1 / 2$ & 1 & 1.0 & 10.0 & 0.15 & 10.0 & 0.128 & 8.8 \\
\hline $63.9419 \pm 3 \times 10^{-4 a}$ & $1 / 2$ & 1 & 0.70 & 10.0 & 0.16 & 10. & 0.133 & 8.3 \\
\hline $64.95 \pm 6 \times 10^{-2}$ & $1 / 2$ & 1 & 127. & 10.0 & 0.079 & 9.9 & 0.079 & 9.9 \\
\hline $65.18 \pm 2 \times 10^{-2} \mathrm{a}$ & $3 / 2$ & 1 & 129.0 & 9.7 & 0.47 & 7.8 & 0.938 & 7.8 \\
\hline $65.362 \pm 2 \times 10^{-3}$ & $1 / 2$ & 0 & 0.70 & 10.0 & 0.30 & 10. & 0.212 & 7.6 \\
\hline $68.591 \pm 7 \times 10^{-3}$ & $3 / 2$ & 1 & 5.5 & 9.3 & 0.43 & 9.8 & 0.802 & 9.1 \\
\hline
\end{tabular}

a New.

${ }^{b}$ Doublet, identified as a single resonance in previous measurements $[14,15]$.

acquisition techniques with fast digitizers, which allowed us to analyze the data off-line in the most flexible way, including an efficient pulse shape analysis for $n / \gamma$ discrimination. Other developments in favor of the present data are related to the very low neutron sensitivity of the n_TOF setup and the use of the well tested $R$-matrix code [30].
In this context, it should be noted that the low-neutroninduced background, obtained with the optimized experimental setup and with the extremely small duty factor of the n_TOF facility, represents a strong improvement in measurements of small capture cross section as in the case of ${ }^{90} \mathrm{Zr}$. 

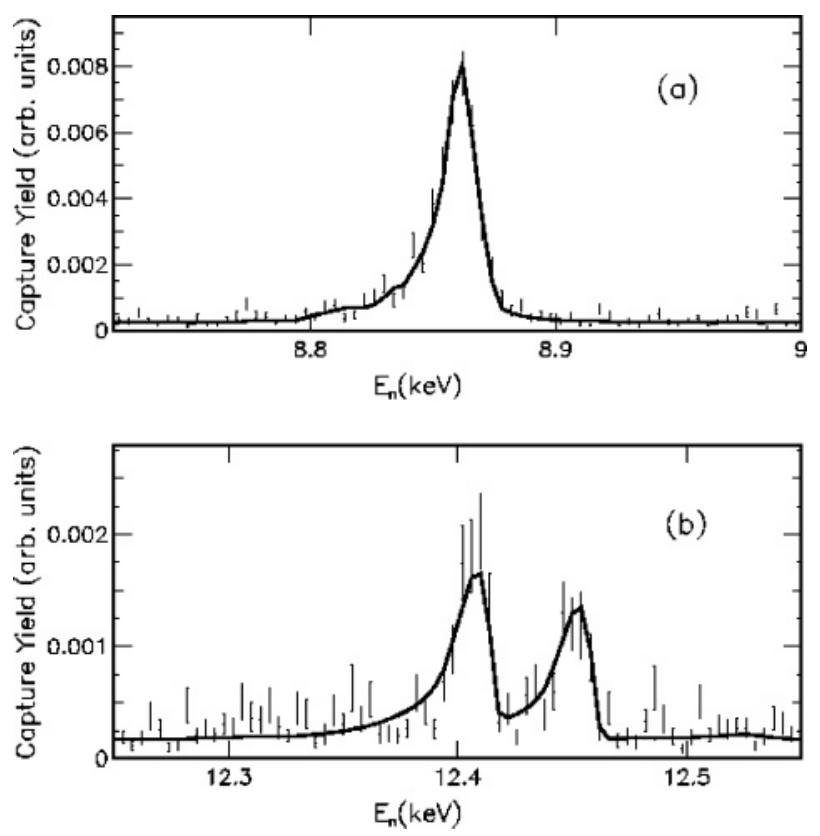

FIG. 3. Capture yield as a function of the energy of incident neutrons (En). Examples for fits with the $R$-matrix code SAMMY for a previously reported resonance (a) and two small resonances, which were observed for the first time (b).

\section{MAXWELLIAN AVERAGED CROSS SECTIONS AND ASTROPHYSICAL IMPLICATIONS}

The calculation of MACS at typical $s$-process temperatures has to be carried out by folding the capture cross section with

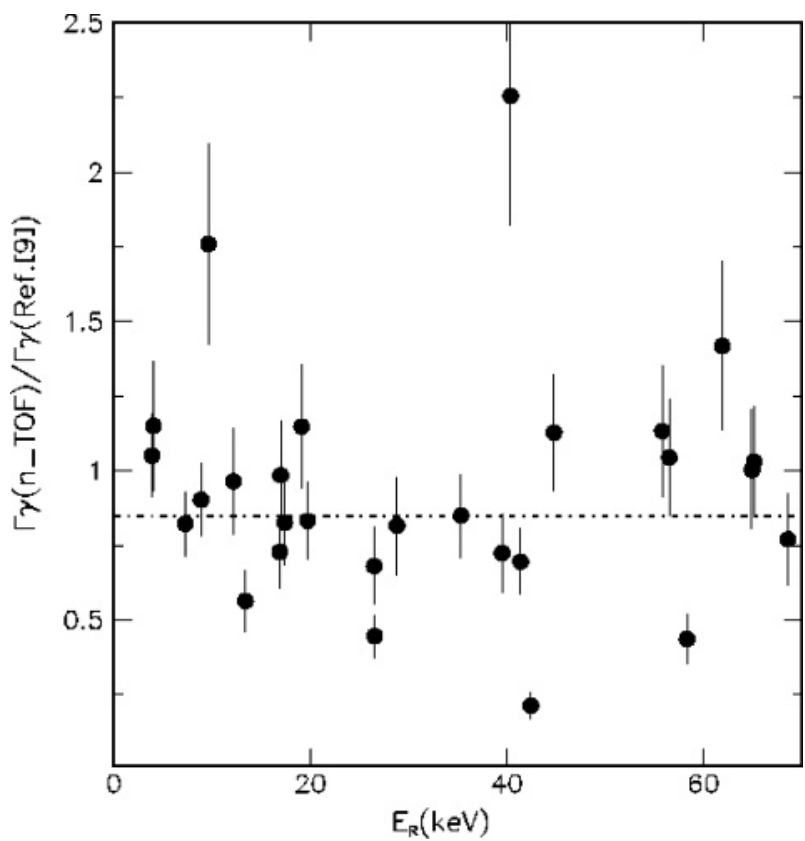

FIG. 4. Ratio between $\Gamma_{\gamma}$ values extracted from the present measurement and the results reported in Ref. [9]. The average is indicated by the dashed-dotted line.
TABLE III. Relative contribution of strong resonances ${ }^{\mathrm{a}}$ (in percentages) to the MACS at thermal energies of $k T=5$ and $30 \mathrm{keV}$.

\begin{tabular}{lcclcc}
\hline \hline \multirow{2}{*}{$E_{R}(\mathrm{keV})$} & \multicolumn{2}{c}{$k T=5 \mathrm{keV}$} & & \multicolumn{2}{c}{$k T=30 \mathrm{keV}$} \\
\cline { 2 - 3 } \cline { 5 - 6 } & Ref. [15] & This work & & Ref. [15] & This work \\
\hline 3.855 & 12.8 & 15.1 & & & \\
4.005 & 25.4 & 24.8 & & & \\
7.251 & 31.1 & 28.5 & & 8.2 & 7.6 \\
8.852 & 15.0 & 15.2 & & 5.1 & 5.3 \\
19.685 & & & & 5.0 & 4.7 \\
26.530 & & & & 5.7 & 5.7 \\
41.340 & & & & 21.1 & 16.7 \\
\hline \hline
\end{tabular}

${ }^{a}$ Only resonances with relative contributions $\geqslant 4 \%$ are considered.

the thermalized stellar spectra over a sufficiently wide neutron energy range, starting at about $100 \mathrm{eV}$ and extending to about $500 \mathrm{keV}$ at the highest temperatures reached during carbon shell burning in massive stars. The $s$-process production occurs predominantly in thermal pulsing low mass AGB stars, where neutrons are released by $(\alpha, n)$ reactions on ${ }^{13} \mathrm{C}$ and ${ }^{22} \mathrm{Ne}$ at effective thermal energies of $k T=8$ and $23 \mathrm{keV}$, respectively. Although this fact restricts the energy range, where the capture cross section has to be known, it nevertheless implies that the present experimental data have to be complemented for the neutron energy range above $70 \mathrm{keV}$.

For the resonance dominated cross section of ${ }^{90} \mathrm{Zr}$ it is obvious that the MACS is strongly dependent on the energy and strength of the most prominent resonances. To illustrate this effect, we evaluated the impact of the strongest resonances for thermal energies of $k T=5$ and $30 \mathrm{keV}$ (Table III). Already the first four resonances account for more than $84 \%$ of the MACS at $k T=5$, whereas the five most important resonances contribute only $45 \%$ at $k T=30 \mathrm{keV}$.

Because $\Gamma_{n} \gg \Gamma_{\gamma}$ in many of the observed resonances, the capture kernels are in first approximation proportional to $\Gamma_{\gamma}$. The comparison in Fig. 5 shows that the kernels obtained in the present analysis are $\approx 15 \%$ smaller than those of Ref. [9]. On average, they are also $10 \%$ weaker than listed in the evaluation of Ref. [14]. Surprisingly, the evaluated kernels have been recently reduced as well, even before new experimental data became available (compare Refs. [14] and [31], for example).

Consequently, the MACS calculated with the present experimental data are up to $10 \%$ lower than those obtained with previous data as shown in Table IV and Fig. 6. Another important improvement of this work is the reduction of uncertainties by more than a factor of 2 .

The impact of the improved MACS for the $s$ abundance of ${ }^{90} \mathrm{Zr}$ refers predominantly to the main $s$ component produced in thermal pulsing low mass AGB stars. The contribution from massive stars is limited to about $2 \%[3,32,33]$; therefore, the new cross section has negligible consequences for the abundance of ${ }^{90} \mathrm{Zr}$ provided by the weak component.

The $s$ abundances produced in thermally pulsing low mass AGB stars has been studied recently by Bisterzo et al. [34], updating previous calculations [5] with an improved stellar model and with a revised nuclear physics input. If the ${ }^{90} \mathrm{Zr}$ capture cross section [1] is replaced in these calculations by the 
TABLE IV. Comparison of MACS (in mb) listed in the compilation of Ref. [1] with evaluated data from JENDL/3.3 [15] and this work.

\begin{tabular}{rccc}
\hline \hline \multirow{2}{*}{$k T(\mathrm{keV})$} & \multicolumn{3}{c}{ Maxwellian averaged cross sections (in mb) } \\
\cline { 2 - 4 } & Ref. [1] & JENDL/3.3 [15] & This work $^{\mathrm{a}}$ \\
\hline 5 & 43 & $47.7 \pm 4.8$ & $44.4 \pm 2.2$ \\
10 & 34 & $34.3 \pm 3.4$ & $31.3 \pm 1.5$ \\
15 & 29 & $28.5 \pm 2.9$ & $25.8 \pm 1.2$ \\
20 & 25 & $25.0 \pm 2.5$ & $22.7 \pm 1.1$ \\
25 & 23 & $22.6 \pm 2.3$ & $20.7 \pm 1.0$ \\
30 & $21 \pm 2$ & $20.8 \pm 2.1$ & $19.3 \pm 0.9$ \\
40 & 18 & $18.2 \pm 1.8$ & $17.1 \pm 0.8$ \\
50 & 17 & $16.3 \pm 1.6$ & $15.5 \pm 0.8$ \\
60 & 16 & $14.9 \pm 1.5$ & $14.3 \pm 0.7$ \\
80 & 15 & $12.8 \pm 1.3$ & $12.4 \pm 0.6$ \\
100 & 14 & $11.3 \pm 1.1$ & $11.0 \pm 0.5$ \\
\hline \hline
\end{tabular}

${ }^{\mathrm{a}}$ Complemented by data from JENDL/3.3 [15] above $70 \mathrm{keV}$.

present result, one obtains an $11 \%$ increase of the contribution from low mass stars, which are now producing $81 \%$ of solar ${ }^{90} \mathrm{Zr}$. Consequently, the $r$-process residual,

$$
N_{r}=N_{\odot}-N_{s}
$$

changed significantly from 25 to $17 \%$. Compared to the $N_{r}$ distribution of the even isotopes in this mass region, this value

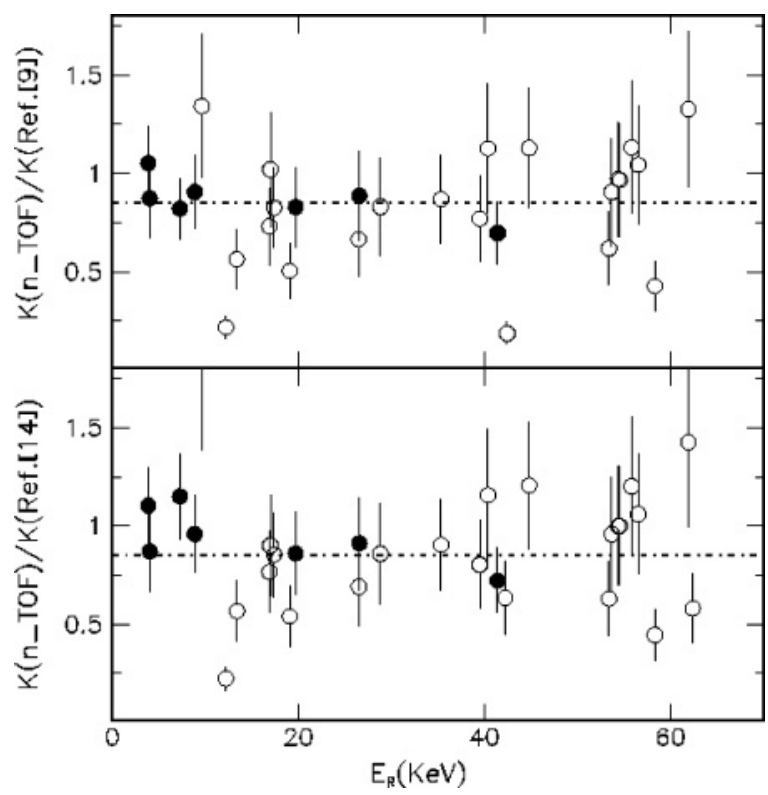

FIG. 5. (Upper panel) Ratio between capture kernels obtained in the present measurement and those of Ref. [9]. (Lower panel) Ratio between capture kernels obtained in the present measurements and those evaluated in Ref. [14]. The strong resonances considered in Table III are indicated by full circles. The average is given by the dashed-dotted line.

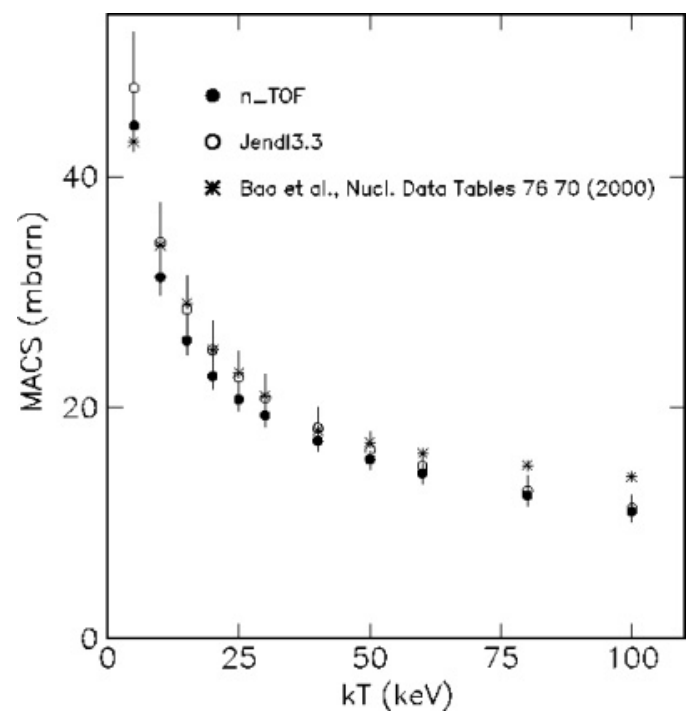

FIG. 6. Comparison of MACSs calculated with present experimental results complemented by data from JENDL/3.3 [15] above $70 \mathrm{keV}$ (full circles) with values obtained exclusively with data from Ref. [15] (open circles) and with the compilation of Ref. [1] (asterisks).

is still higher but compatible within uncertainties as expected for a smooth $r$-process pattern [5].

\section{CONCLUSIONS}

The $(n, \gamma)$ cross section of the neutron magic isotope ${ }^{90} \mathrm{Zr}$ has been measured with improved accuracy over a wide range of neutron energies taking advantage of the unique features of the $\mathrm{n}_{-} \mathrm{TOF}$ facility at CERN. Resonance parameters for 45 resonances (10 newly identified) were determined in the neutron energy range between 3 and $70 \mathrm{keV}$. The capture kernels of the analyzed resonances are significantly smaller than those reported in previous measurements and in evaluated data libraries. This difference can be understood in terms of the outstanding performance of the facility and by improvements of the experimental setup and of the data analysis package. Maxwellian averaged cross sections calculated with the new capture data are up to $10 \%$ smaller than before. This is an important input for studies of $s$-process nucleosynthesis, because ${ }^{90} \mathrm{Zr}$ belongs to the neutron magic nuclei with $N=50$, which represent a bottleneck for the reaction flow toward heavier nuclei. The present result indicates a significantly smaller $r$-process abundance, compatible with a smooth interpolation from neighboring even-even isotopes.

\section{ACKNOWLEDGMENTS}

This work was supported by the EC under contract FIKW-CT-2000-00107 and by the funding agencies of the participating institutes. 
[1] Z. Y. Bao, H. Beer, F. Käppeler, F. Voss, K. Wisshak, and T. Rauscher, At. Data Nucl. Data Tables 76, 70 (2000).

[2] E. Anders and N. Grevesse, Geochim. Cosmochim. Acta 57, 197 (1989).

[3] C. M. Raiteri, R. Gallino, M. Busso, D. Neuberger, and F. Käppeler, Astrophys. J. 419, 207 (1993).

[4] R. Gallino, C. Arlandini, M. Busso, M. Lugaro, C. Travaglio, O. Straniero, A. Chieffi, and M. Limongi, Astrophys. J. 497, 388 (1998).

[5] C. Arlandini, F. Käppeler, K. Wisshak, R. Gallino, M. Lugaro, M. Busso, and O. Straniero, Astrophys. J. 525, 886 (1999).

[6] M. Lugaro, F. Herwig, J. C. Lattanzio, R. Gallino, and O. Straniero, Astrophys. J. 586, 1305 (2003).

[7] M. Lugaro, A. M. Davis, R. Gallino, J. Pellin, O. Straniero, and F. Käppeler, Astrophys. J. 593, 486 (2003).

[8] R. J. Stancliffe, M. Lugaro, A. I. Karakas, and C. A. Tout, Nucl. Phys. A758, 569 (2005).

[9] J. W. Boldeman, B. J. Allen, A. R. de L. Musgrove, and R. L. Macklin, Nucl. Phys. A246, 1 (1975).

[10] R. E. Toohey and H. E. Jackson, Phys. Rev. C 9, 346 (1974).

[11] Z. M. Bartolome, R. W. Hockenbury, W. R. Moyer, J. R. Tatarczuk, and R. C. Block, Nucl. Sci. Eng. 37, 137 (1969).

[12] W. M. Good and H. Kim, Phys. Rev. 165, 1329 (1968).

[13] S. S. Moskalev, H. V. Muradian, and Yu. V. Adamchuk, Nucl. Phys. 53, 667 (1964).

[14] S. F. Mughabghab, Atlas of Neutron Resonances, Resonance Parameters and Thermal Cross Sections $Z=1-100$ (Elsevier Science, Amsterdam, 2006).

[15] For results compiled in evaluated nuclear data libraries see for example International Atomic Energy Agency (IAEA) on www-nds.iaea.org, or the OECD Nuclear Energy Agency on www.nea.fr/html/dbdata/

[16] http://www.nea.fr/html/dbdata/hprl

[17] C. Borcea et al., Nucl. Instrum. Methods A 513, 523 (2003).
[18] U. Abbondanno et al., CERN n_TOF facility: Performance report, CERN-SL-2002-053 ECT (2003).

[19] S. Marrone et al., Phys. Rev. C 73, 034604 (2006).

[20] R. Plag, M. Heil, F. Käppeler, P. Pavlopoulos, R. Reifarth, and K. Wisshak, Nucl. Instrum. Methods A 496, 425 (2003).

[21] U. Abbondanno et al., Nucl. Instrum. Methods A 538, 692 (2005).

[22] S. Marrone et al., Nucl. Instrum. Methods A 517, 389 (2004).

[23] R. L. Macklin and J. Halperin, Phys. Rev. C 14, 1389 (1976).

[24] F. Corvi, G. Fioni, F. Gasperini, and P. B. Smith, Nucl. Sci. Eng. 107, 272 (1991).

[25] J. N. Wilson et al., Nucl. Instrum. Methods A 511, 388 (2003).

[26] U. Abbondanno et al., Nucl. Instrum. Methods A 521, 454 (2004).

[27] G. Aerts et al., Phys. Rev. C 73, 054610 (2006).

[28] The $n_{-}$TOF Collaboration, Report CERN-INTC-O-011, CERN, 2003.

[29] G. Lorusso et al., Nucl. Instrum. Methods A 532, 622 (2004).

[30] N. M. Larson, computer code Report ORNL/TM-9179/R7, 2006.

[31] S. F. Mughabghab, Neutron Cross Sections: Neutron Resonance Parameters and Thermal Cross Sections (Academic Press, New York, 1984).

[32] T. Rauscher, A. Heger, R. D. Hoffman, and S. E. Woosley, Astrophys. J. 576, 323 (2002).

[33] M. Pignatari et al., Nuclei in the Cosmos-IX, Proceedings of Science, SISSA, Trieste, 2006, edited by A. Mengoni et al., http://pos.sissa.it//archive/conferences/028/061/NICIX_061.pdf.

[34] S. Bisterzo et al., Nuclei in the Cosmos-IX, Proceedings of Science, SISSA, Trieste, 2006, edited by A. Mengoni et al., http://pos.sissa.it//archive/conferences/028/077/NICIX_077.pdf. 